\title{
Method for Aerosol Parameter Estimation Error Analysis - Consideration of Noises Included in the Measured Solar Direct and Diffuse Irradiance
}

\author{
Kohei Arai ${ }^{1}$ \\ 1 Graduate School of Science and Engineering \\ Saga University \\ Saga City, Japan
}

\begin{abstract}
Method for error analysis of aerosol parameter estimation with assumed noises which are included in the measured solar direct and diffuse irradiance is proposed. The noises included in the measured solar direct and diffuse irradiance are assumed to be Chi-Square probability density function due to the fact that the measured irradiance is represented as output power which is square of output voltage which corresponds to the measured irradiance which is assumed to be normal distribution of probability density function. Aerosol parameters (refractive index which consists of real and imaginary parts, size distribution which is represented by Junge parameter) are estimated with the measured solar direct and diffuse irradiance which corresponds to the acquired output power of the measuring instrument which allows measurement of solar direct and diffuse irradiance. Through experiments with the measured solar direct and diffuse irradiance, it is found that the estimation accuracy of imaginary part of aerosol refractive index is the most sensitive to the added noises followed by size distribution of Junge parameter and real part of aerosol refractive index.
\end{abstract}

Keywords-Aerosol; Atmospheric optical depth; Solar irradiance; Solar direct; Solar diffuse; Aereole; Junge parameter; Size distribution; Real and imaginary parts of refractive index

\section{INTRODUCTION}

The largest uncertainty in estimation of the effects of atmospheric aerosols on climate systems is from uncertainties in the determination of their microphysical properties, including the aerosol complex index of refraction that in turn determines their optical properties. The methods, which allow estimation of refractive indices, have being proposed so far [1]-[3].

Most of the methods use ground based direct, diffuse and aureole measurement data such as AERONET [4] and SKYNET [5]. The methodology for estimation of a complete set of vertically resolved aerosol size distribution and refractive index data, yielding the vertical distribution of aerosol optical properties required for the determination of aerosol-induced radiative flux changes is proposed [6].

The method based on the optical constants determined from the radiative transfer models of the atmosphere is also proposed [7]. Laboratory based refractive indices estimation methods with spectral extinction measurements are proposed
[8], [9]. All these existing methods are based on radiance from the sun and the atmosphere.

Through atmospheric optical depth measurements with a variety of relatively transparent wavelength, it is possible to estimate size distribution, molecule scattering, gaseous transmission, ozone and water vapor absorptions, etc. so that refractive index might be estimated [10]-[14]. In order to assess the estimation accuracy of refractive index with the proposed method, sensitivity analysis is conducted with a variety of parameters of the atmosphere. In particular, observation angle dependency is critical for atmospheric optical depth measurements. Therefore, it is conducted to assess influences due to observation angle on estimation accuracies of refractive index and size distribution. Similar researches are conducted and well reported [15]-[27].

The next section describes the proposed system followed by experiment. Then concluding remarks are described with some discussions.

\section{PROPOSED METHOD}

\section{A. Radiative Transfer Function}

Measured solar direct irradiance $F$ on the ground is expressed in equation (1)

$$
F=F_{0} e^{-m_{0} \tau_{i}}
$$

where $F_{0}$ denotes extraterrestrial solar flux, $m_{0}$ denotes airmass which can be represented as $1 / \cos (\theta)$ where $\Theta$ denotes solar zenith angle, and $\tau_{t}$ denotes atmospheric optical depth which can be expressed in equation (2)

$$
\tau_{t}=\tau_{a}+\tau_{m}=\tau_{a s}+\tau_{a a}+\tau_{m s}+\tau_{m a}
$$

where the first suffix $t, a$ and $m$ denotes total atmosphere, aerosols and molecules, respectively while the second suffix $a$ and $s$ denotes absorption and scattering, respectively. $F$ can be measured on the ground while $F_{0}$ is well modeled by many researchers. On the other hand, $m_{0}$ can be well determined which results in estimation of atmospheric optical depth. Atmospheric optical depth due to aerosol and molecule has to be estimated together with their absorption and scattering components.

Meanwhile, measured solar diffuse irradiance on the ground can be expressed in equation (3). 


$$
E\left(\theta_{0}, \varphi\right)=E(\vartheta)=F m_{0} \Delta \Omega\left\{\omega \tau_{t} P(\vartheta)+q(\vartheta)\right\}
$$

where $\varphi$ denotes the angle between solar azimuth and observation azimuth directions while $\vartheta$ denotes azimuth and elevation angles. There is the following relation between both angles,

$$
\cos (\vartheta)=\cos ^{2} \theta_{0}+\sin ^{2} \theta_{0} \cos \varphi
$$

$\Delta \Omega$ denotes solid angle of the solar diffuse measuring instrument while $\omega$ denotes single scattering albedo which can be represented as follows,

$$
\omega=\left(\tau_{a s}+\tau_{m s}\right) / \tau_{t_{k}}
$$

$P(\vartheta)$ and $q(\vartheta)$ denotes scattering phase function and multiple scattering component, respectively. $P(\vartheta)$ is expressed as follows,

$$
P(\vartheta)=\left\{\tau_{m s} P_{m}(\vartheta)+\tau_{a s} P_{a}(\vartheta)\right\} /\left(\tau_{m s}+\tau_{a s}\right)
$$

Because of the observation wavelength and molecule radius has the following relation,

$$
\frac{\pi r_{a}}{\lambda}<0.4
$$

molecule component of scattering can be expressed based on the Rayleigh scattering theory. Molecule component of scattering phase function $P_{m}(\vartheta)$ can be expressed as follows,

$$
P_{m}(\vartheta)=\left(\frac{3}{4}\right)\left(1+\cos ^{2} \vartheta\right)
$$

Molecule scattering component of the atmospheric optical depth is represented as follows,

$\tau_{m s}=$

$$
\left\{0.008569 \lambda^{-4}\left(1+0.0113 \lambda^{-2}+0.00013 \lambda^{-4}\right)\right\}\left(\frac{p}{p_{0}}\right)\left(\frac{T_{0}}{T}\right)
$$

where $\lambda$ denotes observation wavelength while $p$ and $p_{0}$ denotes atmospheric pressure on the ground, standard atmospheric pressure $(1013.25 \mathrm{hPa})$, respectively. On the other hand, $T_{0}$ and $T$ denotes standard air-temperature on the ground $(288.15 \mathrm{~K})$ and air-temperature on the ground, respectively.

Meanwhile, observation wavelength and aerosol particle size has the following relation,

$$
0.4<\frac{\pi r_{a}}{\lambda}<3
$$
theory.

Aerosol scattering is expressed based on the Mie scattering

Aerosol scattering intensity is expressed as equation (11).

$$
\beta_{a}(\vartheta)=\frac{r^{2}}{2 \pi} \int_{r_{\min }}^{r_{\max }}\left\{i_{1}(\vartheta, x, \tilde{m})+i_{2}(\vartheta, x, \tilde{m})\right\} n(r) d r
$$

where $i_{1}, i_{2}$ denotes Mie scattering intensity function as the function of $x$ of size parameter, $\vartheta$, and $\tilde{m}$ of aerosol refractive index. On the other hand, $n(r)$ denotes the number of aerosol particles of which the radius is $r$ and is called as number of aerosol particle size distribution in unit of $1 / \mathrm{cm}^{2} / \mu \mathrm{m}$.

$$
\mathrm{n}(\mathrm{r})=\mathrm{dN}(\mathrm{r}) / \mathrm{dr}
$$

The size parameter can be represented as follows,

$$
\mathrm{x}=\left(\frac{2 \pi}{\lambda}\right) r
$$

On the other hand, aerosol optical depth is represented as follows,

$$
\tau_{a}=\int_{r_{\min }}^{r_{\max }} \pi r^{2} Q_{\text {ext }}(x, \widetilde{m}) n(r) d r
$$

where $\mathrm{Q}$ is called as Extinction Efficiency Factor. Sometime, the following volume scattering size distribution is used.

$$
\mathrm{v}(\mathrm{r})=\frac{v d}{d \ln r}
$$

There is the well-known relation between the number and volume of size distributions as follows,

$$
\mathrm{v}(\mathrm{r})=\frac{4}{3} \pi r^{4} n(r)
$$

Junge proposed the following size distribution function with Junge parameter $\gamma$,

$$
\mathrm{Cr}^{-\gamma}=\frac{d n}{d \ln r}
$$

In this paper, the Junge function of size distribution is used because of its simplicity with only one Junge parameter.

Let integral kernel functions be

$$
\begin{aligned}
& K_{\text {ext }}(x, \widetilde{m})=\left(\frac{3}{4}\right) \frac{Q_{\text {ext }}(x, \tilde{m})}{x} \\
& \mathrm{~K}(\vartheta, \mathrm{x}, \widetilde{m})=\frac{3}{2} \frac{i_{1}(\vartheta, x, \tilde{m})+i_{2}(\vartheta, x, \tilde{m})}{x^{3}}
\end{aligned}
$$

Then

$$
\begin{aligned}
& \beta_{a}(\vartheta)=\frac{2 \pi}{\lambda} \int_{r_{\min }}^{r_{\max }} K(\vartheta, x, \widetilde{m}) v(r) d \ln r \\
& \tau_{a}=\frac{2 \pi}{\lambda} \int_{r_{\min }}^{r_{\max }} K_{\text {ext }}(x, \tilde{m}) v(r) d \ln r \\
& P_{a}(\vartheta)=\beta_{a}(\vartheta) / \omega_{a} \tau_{a}
\end{aligned}
$$

Solar diffuse irradiance taking into account the multiple scattering in the atmosphere measured on the ground can be represented as follows,

$$
\begin{aligned}
& \mathrm{L}(\vartheta)=F_{0} m_{0} e^{-m \tau_{t}}\left\{\left(\tau_{m s}+\tau_{M S}\right) P_{m}(\vartheta)+\tau_{a s} P_{a}(\vartheta)+\right. \\
& \left.\tau_{A} P_{m}\left(0^{\circ}\right)\right\}
\end{aligned}
$$

where $\left(\tau_{m s} P_{m}(\vartheta)\right)$ implies Rayleigh scattering component while $\left(\tau_{M S}\right) P_{m}(\vartheta)$ implies multiple scattering component in the atmosphere. On the other hand, $\tau_{a s} P_{a}(\vartheta)$ implies aerosol scattering component while $\tau_{A} P_{m}\left(0^{\circ}\right)$ implies multiple scattering component in the atmosphere after the reflection on

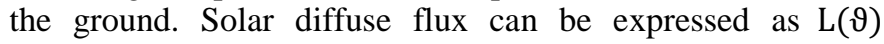
multiplied by observation solid angle $\Delta \Omega$. Meanwhile, $\tau_{M S}$ and $\tau_{A}$ are expressed empirically as follows,

$$
\begin{aligned}
& \tau_{M S}=0.02 \tau_{S S}+1.2 \tau_{S S}^{2} \mu_{0}^{\frac{-1}{4}} \\
& \tau_{A}=\frac{A \tau_{2}}{1-A \tau_{3}}
\end{aligned}
$$

where

$$
\begin{gathered}
\tau_{S S}=\tau_{m s}+\tau_{s a} \\
\mu_{0}=\cos \left(\theta_{0}\right) \\
\tau_{2}=1.34 \tau_{S S} \mu_{0}\left\{1+0.22\left(\frac{\tau_{S S}}{\mu_{0}}\right)^{2}\right\} \\
\tau_{3}=0.9 \tau_{S}-0.92 \tau_{S S}^{2}+0.54 \tau_{S S}^{3}
\end{gathered}
$$

Therefore, the contribution of multiple scattering in the atmosphere is expressed as follows,

$$
\mathrm{q}(\vartheta)=\tau_{M S} P_{m}(\vartheta)+\tau_{A} P_{m}\left(0^{\circ}\right)
$$

\section{B. Actual Radiative Transfer Equation Solving}

The following much stable parameter is introduced, 


$$
R(\vartheta)=\frac{E(\vartheta)}{F m_{0} \Delta \Omega}=\omega \tau_{t} P(\vartheta)+q(\vartheta)=\beta(\vartheta)+q(\vartheta)
$$

Instead of $E(\vartheta), R(\vartheta)$ does not have large influence due to calibration error of the measuring instrument for solar direct and diffuse irradiance. $\omega \tau_{t} P(\vartheta)$ is replaced to $\beta(\vartheta)$. It is called single scattering intensity. Widely used aerosol parameter estimation method and software code is called Skyrad.Pack developed by Teruyuki Nakajima [11]. In the Skyrad.Pack ver.4.2, iteration method is used as follows,

$$
\begin{gathered}
\beta_{a}^{(1)}(\vartheta)=R_{\text {mean }}(\vartheta) \\
\beta_{a}^{(n+1)}(\vartheta)=R_{\text {mean }}(\vartheta) \beta_{a}^{(n)}(\vartheta) /_{R^{(n)}}(\vartheta)
\end{gathered}
$$

where (n) denotes the iteration number while $R_{\text {mean }}(\vartheta)$ denotes the measured solar diffuse irradiance. This method is appropriate in the sense of optimization of single scattering albedo and flux, as well as contribution factor of the multiple scattering component. In order to estimated single scattering flux, we have to know aerosol refractive index and size distribution. Therefore, inverse problem solving method is needed for this. The proposed method uses Moore-Penrose generalized inverse matrix method. Volume vector $v(\mathrm{r}$ dimension) of unknown size distribution $v^{(n)}(r)$ is assumed to be the matrix $g$ which consists of a measured aerosol scattering flux $\beta_{a}^{(n)}(\vartheta)$ and aerosol optical depth $\tau_{a}^{(n-1)}$. Then,

$$
g=G v+\varepsilon
$$

where $G$ denotes a linear multiple term matrix. Thus, the size distribution can be determined as follows,

$$
v=\left(G^{T} G+\eta H\right)^{-1} A^{T} g
$$

where $\mathrm{H}$ denotes a smoothing matrix while $\eta$ denotes Lagrange multiplier.

\section{Added Noise on the Measured Solar Direct and Diffuse} Irradiance

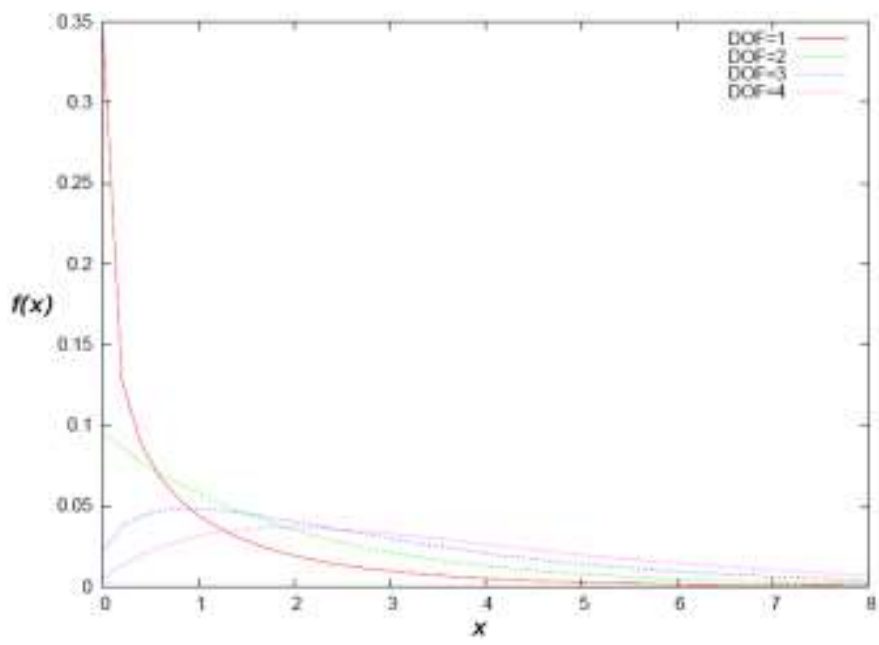

Fig. 1. Examples of the Chi-Square probability density function

The output of the measuring instrument for solar direct and diffuse irradiance is represented as a power. The probability density function of the output noise voltage " $x$ " of the instrument is assumed to be Normal distribution (Gaussian distribution). Therefore, the probability density function of the output noise power " $x$ " of the instrument is assumed to be Chi-Square distribution. Examples of the Chi-Square probability density function $f(x)$ are shown in Fig.1.

Where DOF denotes Degree of Freedom of the parameter of Chi-Square probability density function.

\section{EXPERIMENTS}

\section{A. The Instrument and Data Used}

POM-01 of sky-radiometer which allows measurements of solar direct and diffuse as well as aureole irradiance measurements is used. Fig. 2 shows outlook and calibration coefficient as well as output power trends of the POM-01. POM-01 is set up on the top of the 7th building of the Science and Engineering Faculty of Saga University (1 Honjo, Saga, 840-8502 Japan). POM-01 measures solar direct irradiance with sun tracking capability and solar diffuse irradiance with 50 different diffuse angles in maximum with the following 7 center wavelength, 315, 400, 500, 675, 870, 940, $1020 \mathrm{~nm}$. $315 \mathrm{~nm}$ is ozone absorption band while $940 \mathrm{~nm}$ is water absorption band, respectively.

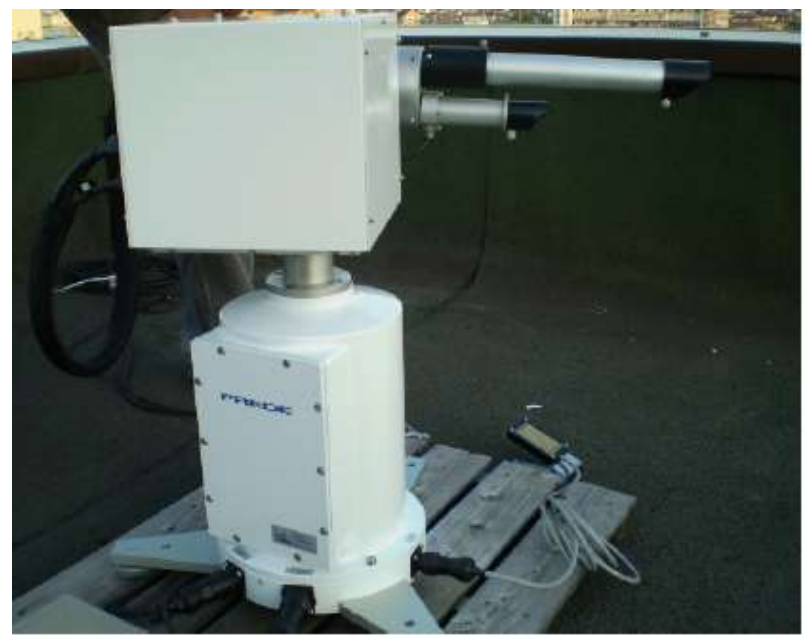

(a) Outlook

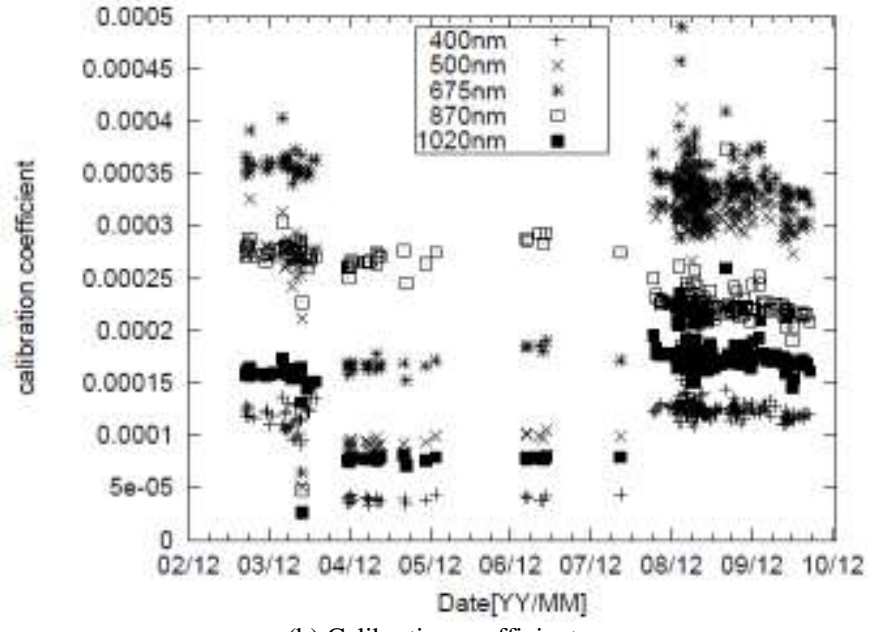

(b) Calibration coefficient 


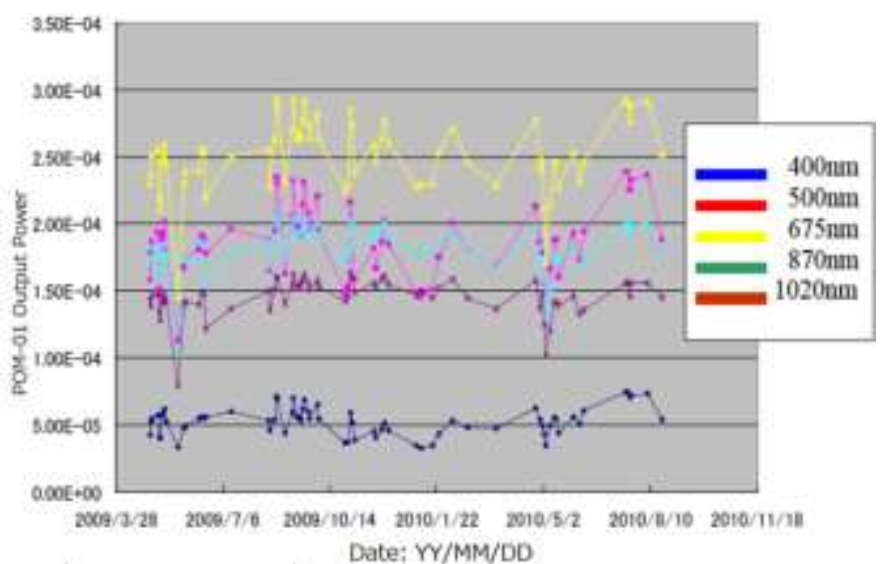

(c) Output power

Fig. 2. Outlook and calibration coefficient as well as output power trend of POM-01

POM-01 has self-calibration function. Using the function, calibration data is acquired routinely. Calibration coefficient trend can be divided into three periods, March 2003 to July 2004, July 2004 to October 2008 and October to now.

Fine weather condition of sky-radiometer data which is measured at 11:08 in the morning on May 252009 in the third period is selected due to the fact that calibration coefficients in the third period are relatively stable.

\section{B. The Preliminary Experiments}

The measured data for both solar direct and diffuse irradiances are the output power of the measuring instrument in unit of [W]. Firstly, the measured output powers are plotted as a function of scattering angle with the different DOF $(1,2,3,4)$ of the additive noise followed by the Chi-Square distribution in Fig.3. In this case, $100 \%$ of noise is added on the measured output power. In accordance with increasing of DOF, the output power of POM-01 is getting up as shown in Fig.3 (a). Meanwhile, the output power of POM-01 decreases in accordance with increasing of DOF as shown in Fig.3 (b).

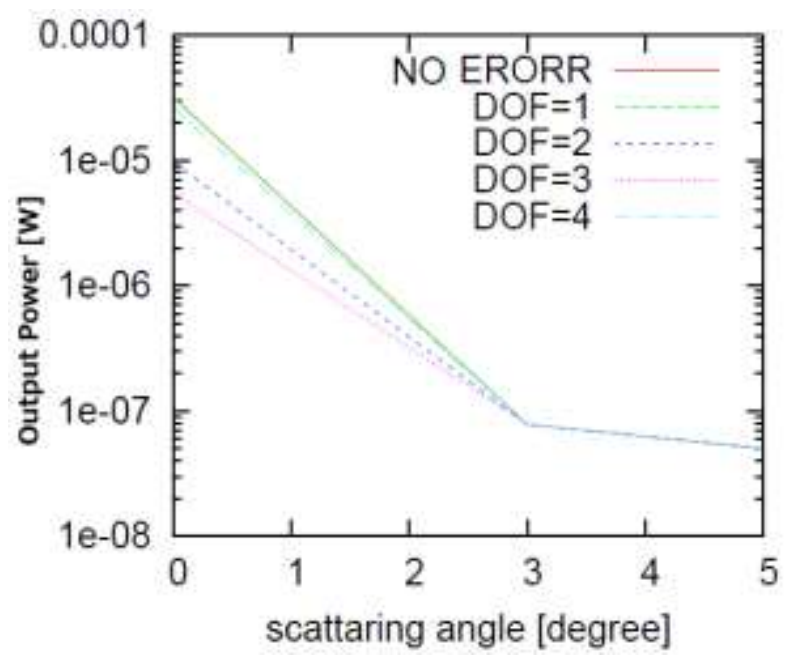

(a) Solar direct

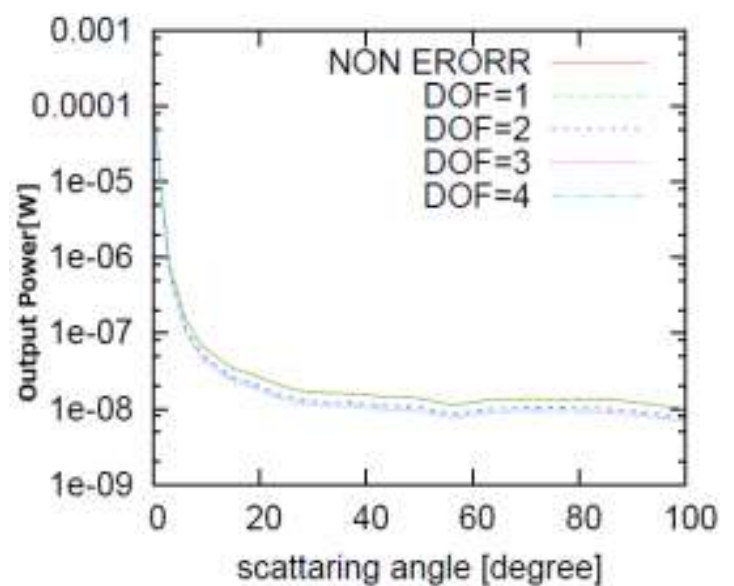

(b) Solar diffuse

Fig. 3. Influence due to Chi-Square distributed additive noise on the output power of the measuring instrument for solar direct and diffuse irradiance

On the other hand, skyrad.pack ver.4.2 allows estimation of volume spectral aerosol size distribution function. Using the relation between volume spectra and Junge size distribution function, equation (16), Junge parameter can be estimated based on the well-known least square method. Fig.4 shows the estimated Junge size distribution function for the aerosols on May 25 2009. It is found that the least square method does works for conversion from volume spectra to Junge distribution function with quit small error.

The skyrad.Pack ver.4.2 requires the parameter for conversion, the number of iterations (NLOOP). In order to determine the parameter, the residual error is calculated as a function of NLOOP for the data which is acquired on May 25 2009. Fig.5 shows the result. Fig.5 also shows the approximate function of residual errors which is expressed with the following function,

$$
f(x)=a x^{b}
$$

where $a=397.708$ and $b=-1.602$.

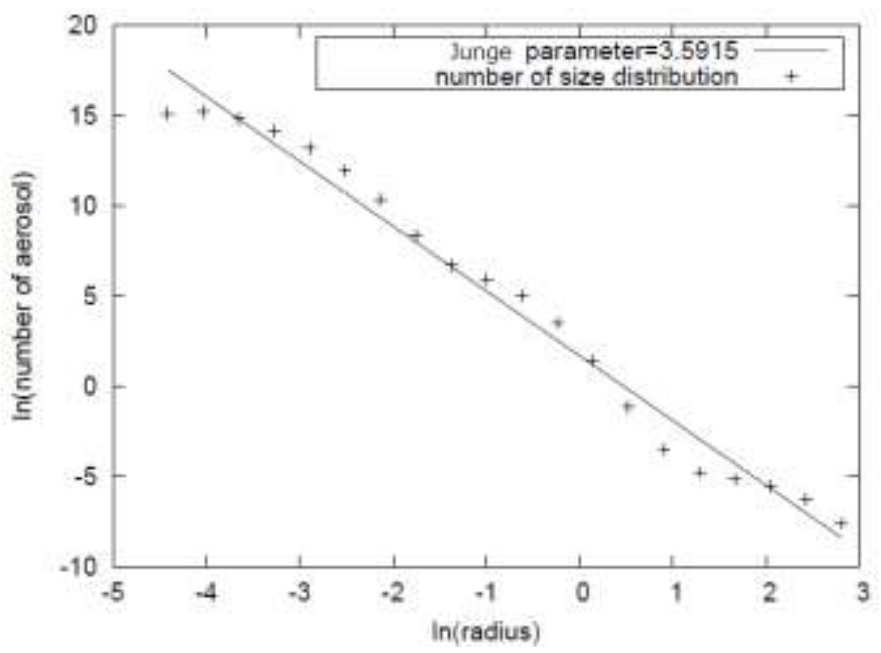

Fig. 4. Junge size distribution function of the aerosols on May 252009 


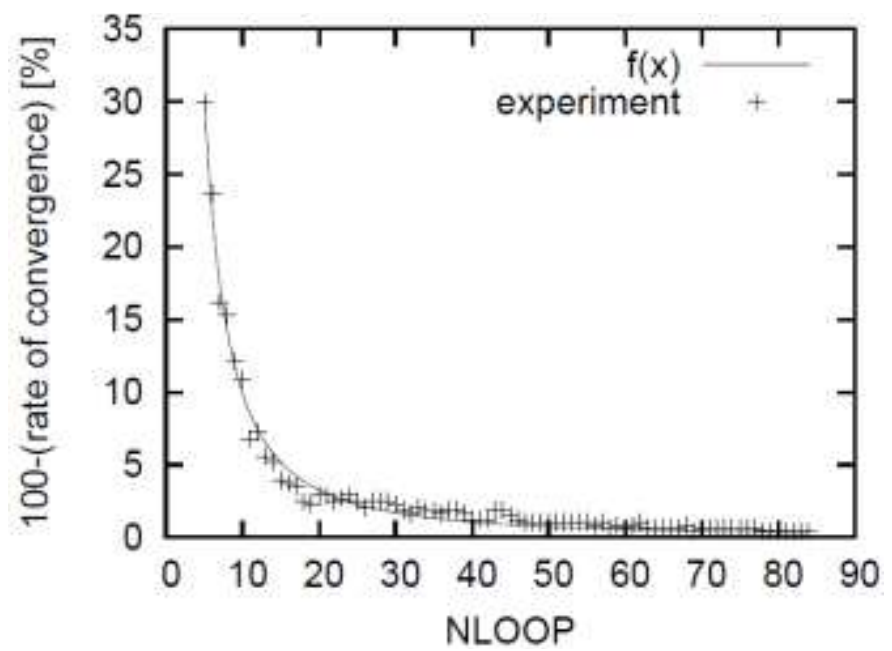

Fig. 5. Residual error (100-rate of conversgence) as the function of the number of iterations (NLOOP)

\section{The Experimental Results}

Using the modified skyrad.pack ver.4.2 described above, aerosol parameters, Real and Imaginary parts of aerosol refractive index and size distribution (Junge parameter) are estimated with the measured solar direct and diffuse irradiance which are measured with POM-01 on May 25 2009. Some of the errors are added on the solar direct angle and solar diffuse angle, respectively. On the other hand, Chi-Square distributed noises are added on the output power of the instrument. Thus sensitivities of the pointing angle error and added noise on the estimated aerosol parameters are clarified.

Fig.6 (a), (b) and (c) shows the solar direct pointing angle error on the estimated real part, imaginary part of refractive index and Junge parameter, respectively. The estimation error is evaluated with percent errors. As shown in Fig.6, it is easily found that the percent errors increase in accordance with increasing of solar direct pointing angle error. Also, it is found that the percent errors increase in accordance with decreasing of wavelength. Meanwhile, added noise influences are not so clear for the changing DOF of the Chi-Square distribution. Because of the influence due to the changing of DOF is not so clear in the Fig.6, two dimensional graphs are created. Fig.7 (a), (b), (c) and (d) shows the influence due to solar direct pointing angle error for the DOF is 1 to 4 on the estimated real part. The estimation error is evaluated with percent errors.

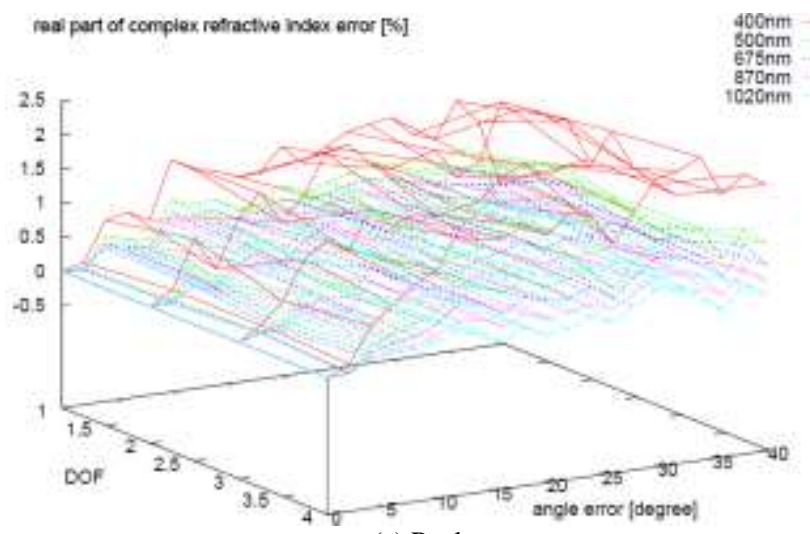

(a) Real part

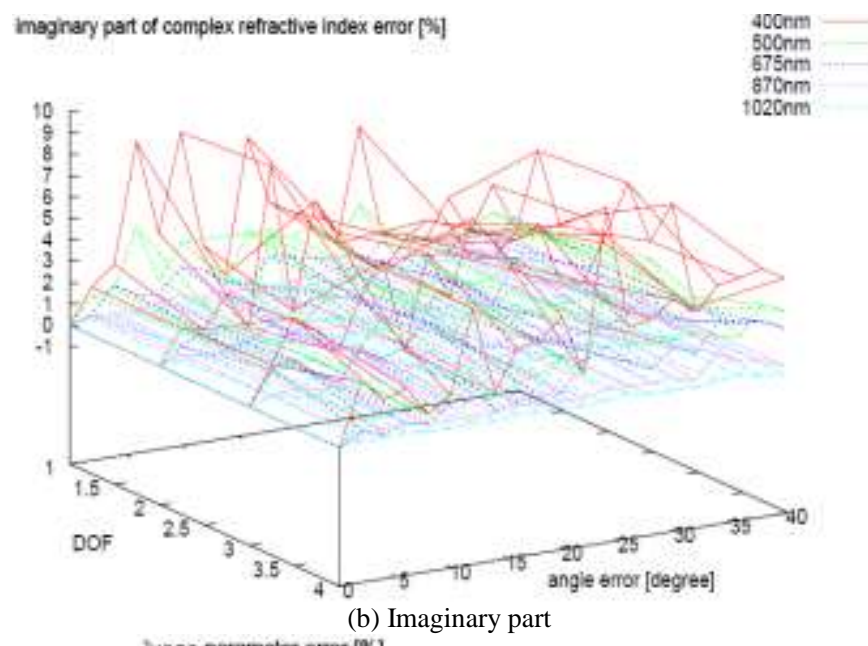

Junge parameter enco $[\%]$

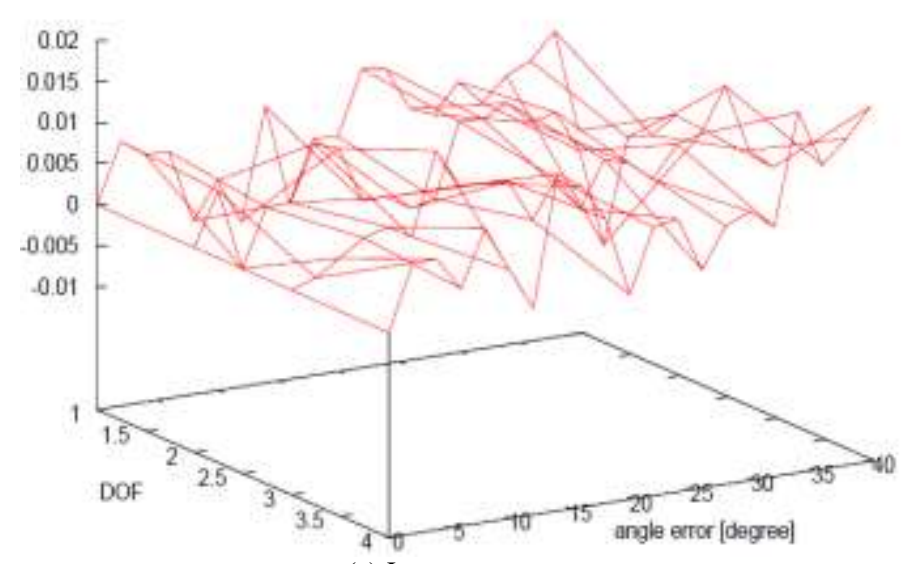

(c) Junge parameter

Fig. 6. Solar direct pointing angle error on the estimated real part, imaginary part of refractive index and Junge parameter

As shown in Fig.7, it is easily found that the percent errors increase in accordance with increasing of solar direct pointing angle error. Also, it is found that the percent errors increase in accordance with decreasing of wavelength.

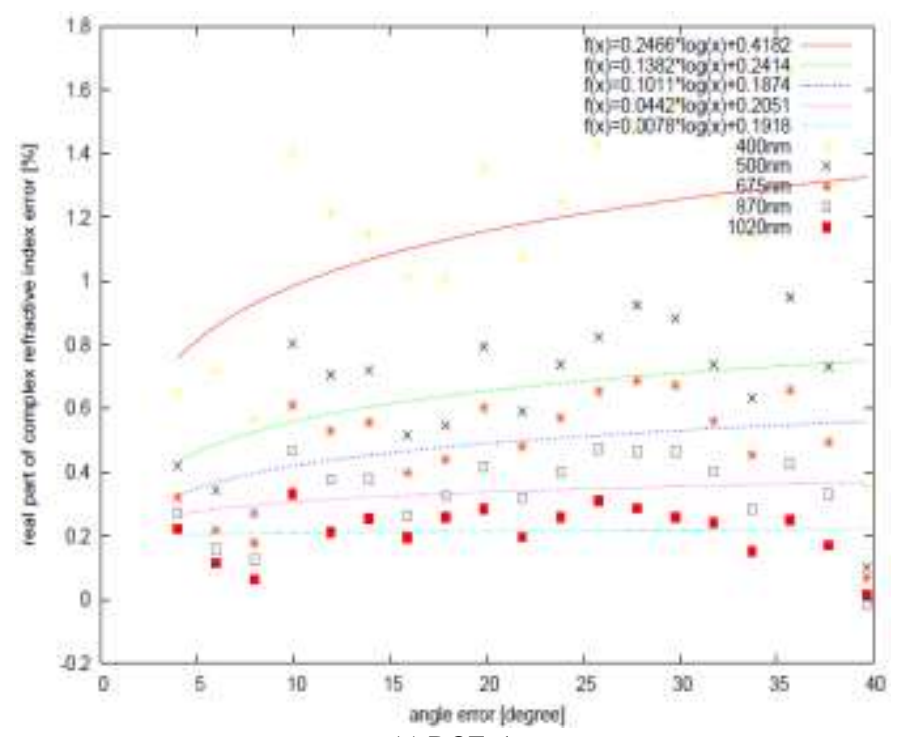

(a) $\mathrm{DOF}=1$ 


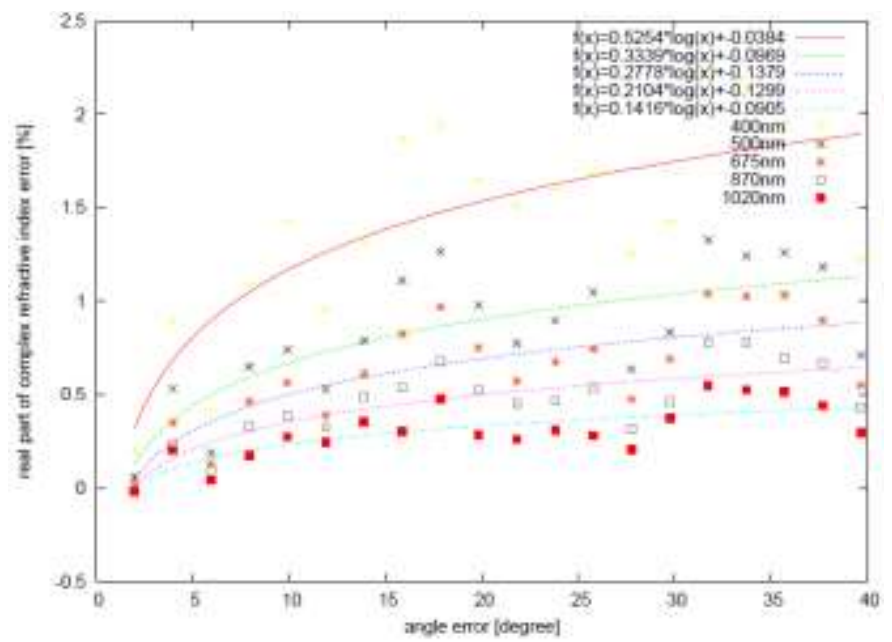

(b) $\mathrm{DOF}=2$

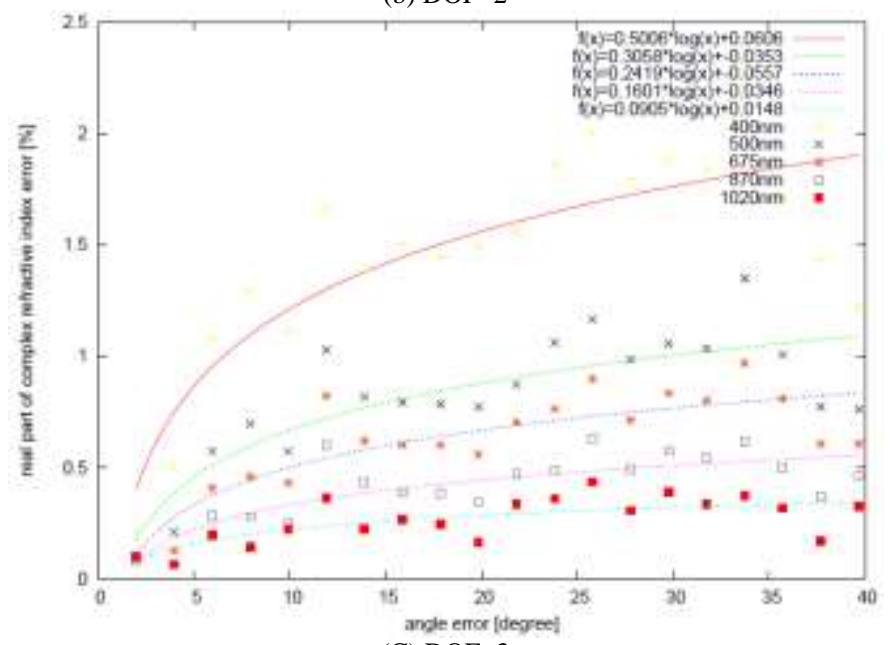

(C) $\mathrm{DOF}=3$

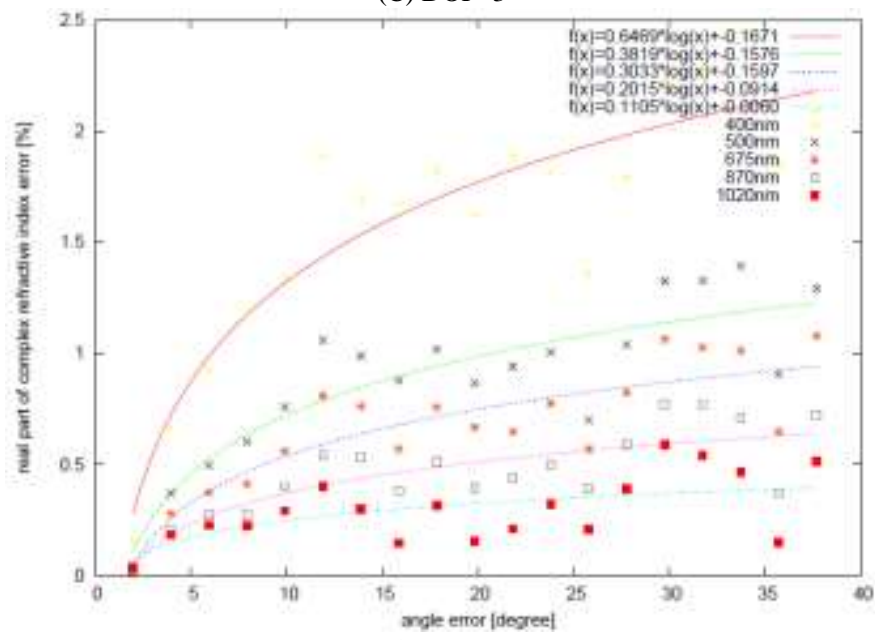

(d) $\mathrm{DOF}=4$

Fig. 7. Influence due to solar direct pointing angle error for the DOF is 1 to 4 on the estimated real part

Fig.8 (a), (b) and (c) shows the solar diffuse pointing angle error on the estimated real part, imaginary part of refractive index and Junge parameter, respectively. The estimation error is evaluated with percent errors. As shown in Fig.8, it is easily found that the percent errors increase in accordance with increasing of solar direct pointing angle error. Also, it is found that the percent errors increase in accordance with decreasing of wavelength. Meanwhile, added noise influences are not so clear for the changing DOF of the Chi-Square distribution.

Because of the influence due to the changing of DOF is not so clear in the Fig.8, two dimensional graphs are created. Fig.9 (a), (b), (c) and (d) shows the influence due to solar diffuse pointing angle error for the DOF is 1 to 4 on the estimated real part. The estimation error is evaluated with percent errors. As shown in Fig.9, it is easily found that the percent errors increase in accordance with increasing of solar diffuse pointing angle error. Also, it is found that the percent errors increase in accordance with decreasing of wavelength.

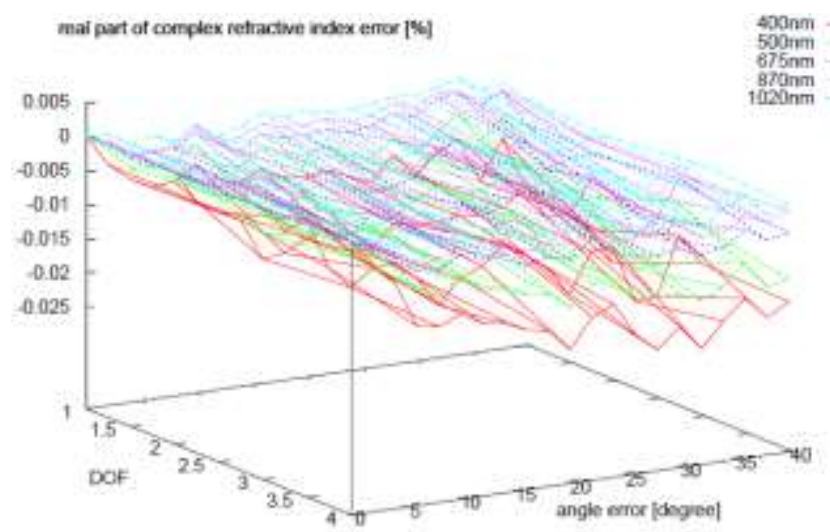

(a) Real part

imaginary part of complex refractive index enor $[\%]$

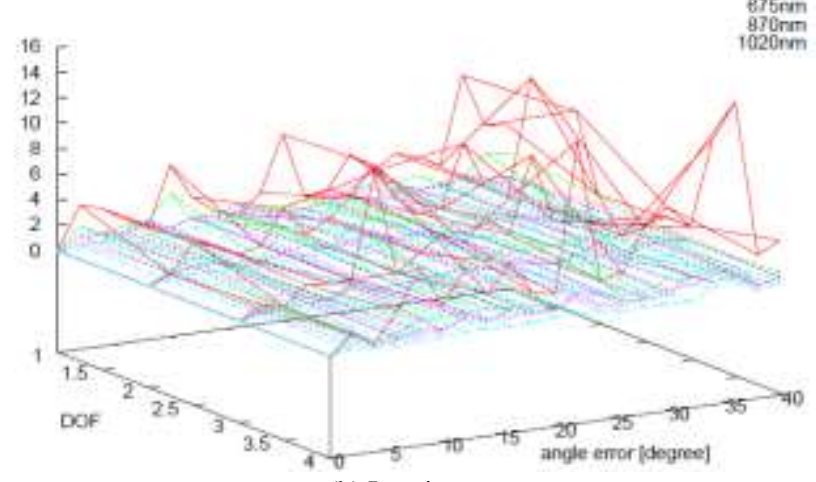

(b) Imaginary part

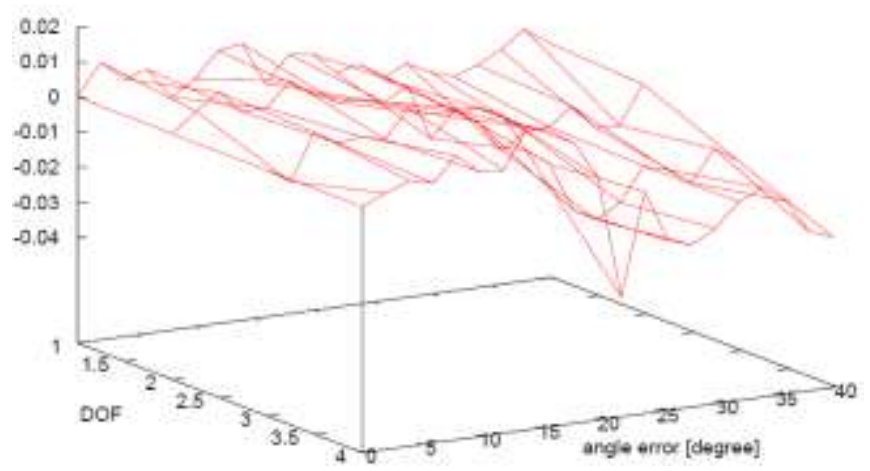

(c) Junge parameter

Fig. 8. Solar diffuse pointing angle error on the estimated real part, imaginary part of refractive index and Junge parameter 


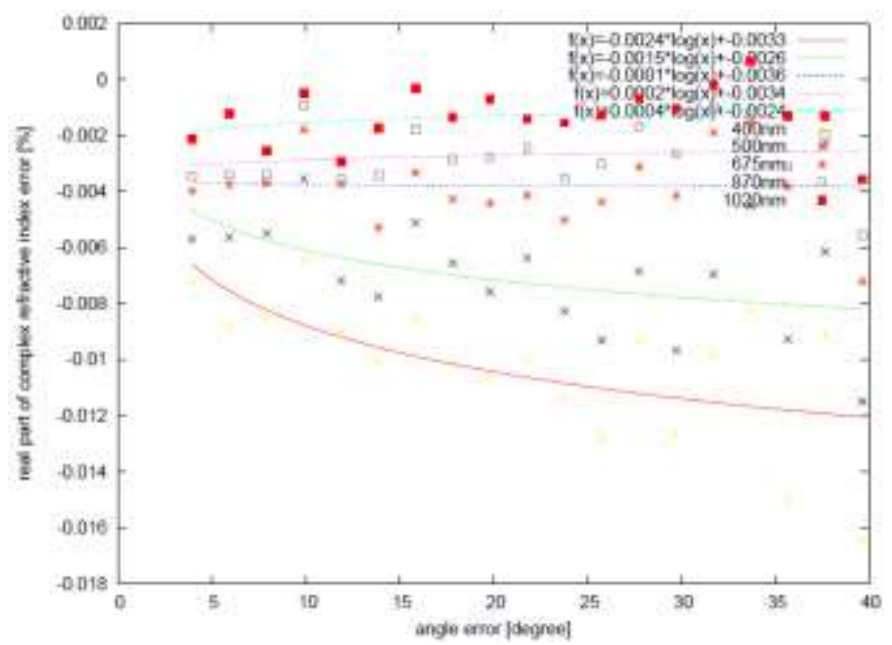

(a) $\mathrm{DOF}=1$

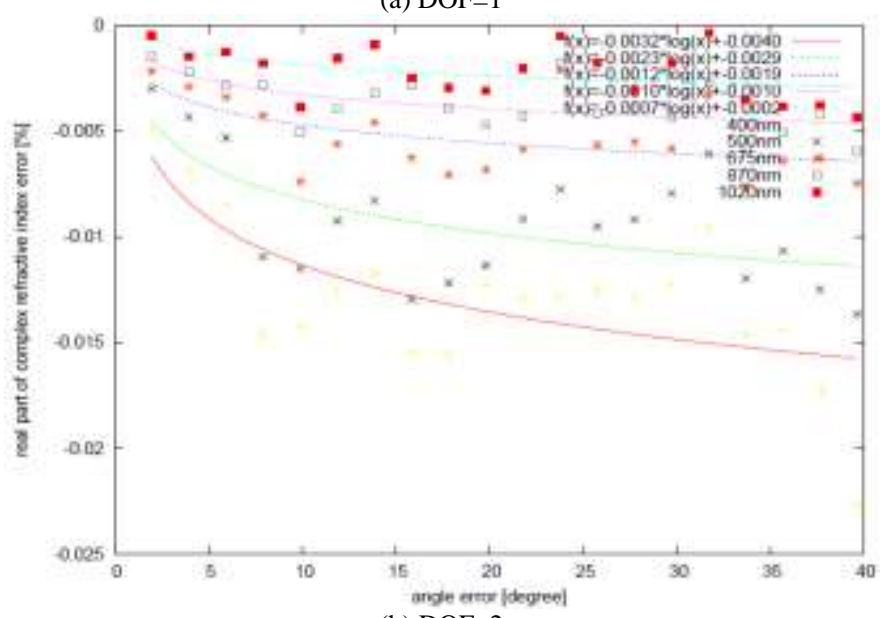

(b) $\mathrm{DOF}=2$

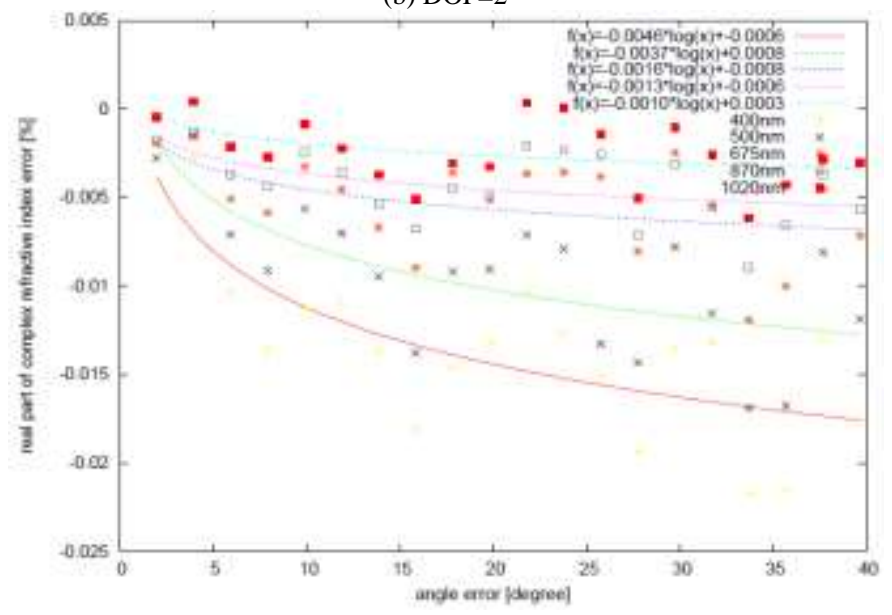

(c) $\mathrm{DOF}=3$

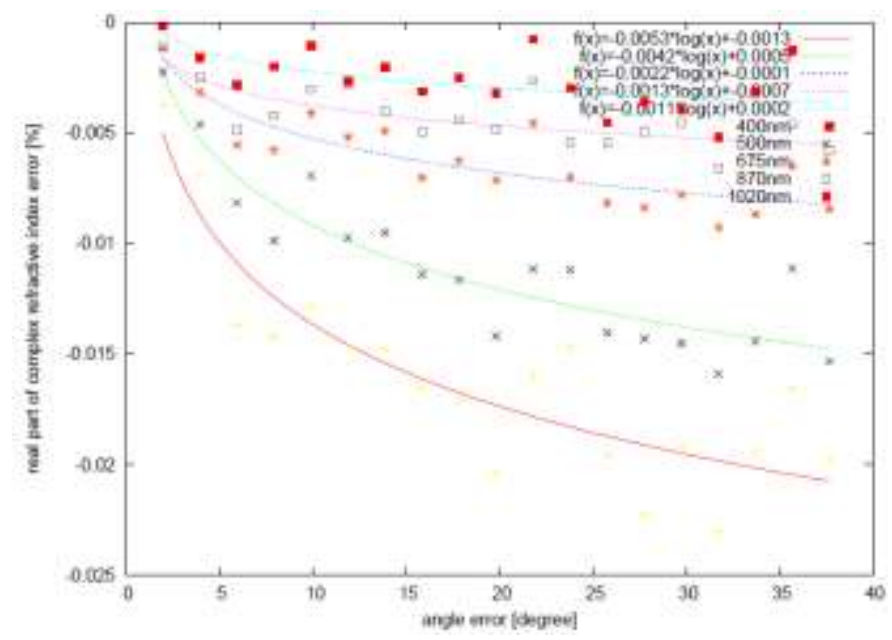

(d) $\mathrm{DOF}=4$

Fig. 9. Influence due to solar diffuse pointing angle error for the DOF is 1 to 4 on the estimated real part

\section{CONCLUSION}

Through the experiments for investigation of influence due to Chi-Square distributed additive noises included in the measured solar direct and diffuse irradiance on estimations of aerosol refractive index and size distribution, it is found that the influence is getting increased according to shortening of the wavelength and is decreasing in accordance with increasing of DOF of Chi-Square probability density function.

When the Chi-Square noises are added to the solar direct irradiance, the estimation error of real part of refractive index is getting large in accordance with increasing of solar direct pointing angle errors because of aerosol scattering factor is over estimated. Also, the estimation error of imaginary part of refractive index is increased with increasing of solar direct pointing angle errors. The influence on imaginary part of refractive index is much significant in comparison to the influence of real part of refractive index due to the fact that the absolute value of the imaginary part of refractive index is much smaller than that of real part of refractive index. The estimation error of real part of refractive index is well approximated with solar direct pointing angle error. All these influence is increased with shortening of the measuring wavelength due to the fact that the influence depends on aerosol optical depth (aerosol optical depth is increased with shortening of the measured wavelength. On the other hand, the influence of solar direct pointing angle error does not depend on the Chi-Square distributed noises of the solar direct irradiance. This is because that there is less sensitive to the solar direct irradiance. 
The estimation error of imaginary refractive index is increased with increasing of the solar diffuse pointing angle error. This is because that aerosol scattering factor is underestimated due to decreasing of solar diffuse irradiance. Also, the estimation error of Junge parameter is decreased with increasing of solar diffuse pointing angle error. The influence of imaginary part of refractive index is greater than that of real part of refractive index due to the fact that size distribution is much sensitive to imaginary part of refractive index comparing to that of real part of refractive index.

Meanwhile, it is found that overall RMSE of real part of refractive index estimations over the experimental conditions is 0.0261 in maximum while that of imaginary part of refractive index is 0.00919 in maximum. On the other hand, overall RMSE of aerosol size distribution of Junge parameter estimations over the experimental conditions is 0.230 in maximum.

The proposed method for error analysis on aerosol parameter estimation influenced by existing noise included in the measured solar direct and diffuse irradiance which is accordance with Chi square probability density function is validated. Further investigation is required for appropriateness of the assumed Chi square probability density function of included noise.

\section{ACKNOWLEDGEMENTS}

Author would like to thank Mr. Yu Odeishi of former student of Saga University for his effort for conducting the experiments.

\section{REFERENCES}

[1] Shaw, G.E., Error analysis of multi-wavelength sunphotometry. Pure Appl. Geophys., 114, 1, 1976.

[2] Hoppel, W. A., J. W. Fitzgerald, G. M. Frick, R. E. Larson, and E. J. Mack, Aerosol size distributions and optical properties found in the marine boundary layer over the Atlantic Ocean, J. Geophys. Res., 95, 3659-3686, 1990.

[3] Holben, B. N., et al., AERONET- A federated instrument network and data achieve for aerosol characterization, Remote Sens., 12, 1147-1163, 1991.

[4] Holben, B.N., and Coauthors, AERONET-A federated instrument network and data archive for aerosol characterization. Remote Sens. Environ., 66, 1-16. 1998

[5] Aoki, K., T. Takamura, and T. Nakajima, Aerosol optical properties measured by SKYNET sky radiometer validation network. Proc. of the 2nd EarthCARE Workshop, 133-134, 2005.

[6] Redemann, R. P. Turco, K. N. Liou, P. B. Russell, R. W. Bergstrom, B. Schmid, J. M. Livingston, P. V. Hobbs, W. S. Hartley, S. Ismail, R. A Ferrare, E. V. Browell, Retrieving the vertical structure of the effective aerosol complex index of refraction from a combination of aerosol in situ and remote sensing measurements during TARFOX, J. Geophys. Res., 105, D8, 9949-9970, 2000.

[7] M.L.Clapp, and R.E.Miller, Complex Refractive Indices of Crystalline Hydrazine from Aerosol Extinction Spectra, Icarus, 23, 2, 396-403(8), 1996.

[8] R. Eiden, Determination of the complex index of refraction of spherical aerosol particles, Appl. Opt. 10, 749-757, 1971.

[9] G. E. Thomas, S. F. Bass, R. G. Grainger, and A. Lambert, Retrieval of aerosol refractive index from extinction spectra with a damped harmonic-oscillator band model, Appl. Opt. 44, 1332-1341, 2005.

[10] Kohei Arai, Aerosol refractive index retrievals with atmospheric polarization measuring data, Proceedings of the SPIE, 7461-06, 1-9, 2009
[11] Teruyuki Nakajima, et al., Use of sky brightness measurements from ground for remote sensing of particulate polydispersions, Applied Optics, Vol.35 No..15 pp.2672-2686 May 1996.

[12] Teruyuki Nakajima, et al., Retrieval of the optical properties of aerosols from aureole and extinction data, Applied Optics, Vol.22 No..19 pp.2951-2959 October 1983.

[13] M. A. Box and A. Deepak, An Approximation to Multiple Scattering in the Earth's Atmo-sphere:Almucanter Radiance Formulation, J. Atmos. Sci. Vol38 pp.1037-1048 1981.

[14] Mitsugu Toriumi, Hideaki Takenaka, Tadashi Kato, Toshikazu Hasegawa, Takashi Nakajima, Tamio Takamura and Teruyuki Nakajima: Estimation of Aerosol Optical Thickness by PAR Radiometer, Journal of The Remote Sensing Society of Japan, 30, 2, pp.81-89, 2010 .

[15] Tsutomu Takashima, Kazuhiko Masuda, Kohei Arai, Satoshi Tsuchida, Observation of Optical Property of the Atmosphere in Particular Aerosols at Deserted Areas In USA Using Sun-photometer and Polarization Spectrometer, Journal of Remote Sensing Society of Japan, Vol.20, No.3, pp.47-60, (2000).

[16] Kohei Arai, Influence of Non-Spherical Sea Salt Aerosol Particles Containing Bubbles on the Top of the Atmosphere Radiance, Journal of Remote Sensing Society of Japan, Vol.20, No.5, pp.32-39, (2001).

[17] Kohei Arai, Tatsuya Kawaguchi, Adjucency Effect of Layered Clouds taking Into Account Phase Function of Cloud Particles and MultiLayered Plane Parallel Atmosphere Based on Monte Carlo Method, Journal of Japan Society of Photogrammetry and Remote Sensing, Vol.40, No.6, 2001.

[18] Kohei Arai, Yoko Takamatsu, Influence of Spume Aerosol Particles on the Top of the Atmosphere Radiance, Journal of Remote Sensing Society of Japan, Vol.23, No.4, pp.355-363, 2003.

[19] Kohei Arai, Xing Ming Liang, Estimation of Method for the Top of the Atmosphere Radiance Taking Into Account Polarized Up-welling and Down-welling Radiance, Journal of Japan Society of Photogrammetry and Remote Sensing, 44,3, 4-12,(2005)

[20] K.Arai, Monte Carlo simulation of polarized atmospheric irradiance for determination of refractive index of aerosols, International Journal of Research and Review on Computer Science, 3, 4, 1744-1748, 2012.

[21] K.Arai, Monte Carlo ray tracing based sensitivity analysis of the atmospheric and oceanic parameters on the top of the atmosphere radiance, International Journal of Advanced Computer Science and Applications, 3, 12, 7-13, 2012.

[22] K.Arai, Monte Carlo ray tracing based sensitivity analysis of the atmospheric and oceanic parameters on the top of the atmosphere radiance, International Journal of Advanced Computer Science and Applications, 3, 12, 7-13, 2012.

[23] Kohei Arai, Method for estimation of aerosol parameters based on ground based atmospheric polarization irradiance measurements, International Journal of Advanced Computer Science and Applications, 4, 2, 226-233, 2013.

[24] Kohei Arai, Sensitivity analysis and validation of refractive index estimation method with ground based atmospheric polarized radiance measurement data, International Journal of Advanced Computer Science and Applications, 4, 3, 1-6, 2013.

[25] Kohei Arai, Sensitivity analysis for aerosol refractive index and size distribution estimation methods based on polarized atmospheric irradiance measurements, International Journal of Advanced Research in Artificial Intelligence, 3, 1, 16-23, 2014.

[26] K.Arai, Vicarious calibration data screening method based on variance of surface reflectance and atmospheric optical depth together with cross calibration, International Journal of Advanced Research on Artificial Intelligence, 4, 11, 1-8, 2015.

[27] K.Arai, Kenta Azuma, Method for surface reflectance estimation with MODIS by means of bi-section between MODIS and estimated radiance as well as atmospheric correction with sky-radiometer, International Journal of Advanced Research on Artificial Intelligence, 4, 11, 8-15, 2015. 


\section{AUTHORS PROFILE}

Kohei Arai, He received BS, MS and PhD degrees in 1972, 1974 and 1982, respectively. He was with The Institute for Industrial Science and Technology of the University of Tokyo from April 1974 to December 1978 and also was with National Space Development Agency of Japan from January, 1979 to March, 1990. During from 1985 to 1987, he was with Canada Centre for Remote Sensing as a Post-Doctoral Fellow of National Science and Engineering Research Council of Canada. He moved to Saga University as a Professor in Department of Information Science on April 1990. He was a counselor for the Aeronautics and Space related to the Technology Committee of the Ministry of Science and Technology during from 1998 to 2000. He was a councilor of Saga University for 2002 and 2003. He also was an executive councilor for the Remote Sensing Society of Japan for 2003 to 2005. He is an Adjunct Professor of University of Arizona, USA since 1998. $\mathrm{He}$ also is Vice Chairman of the Commission-A of ICSU/COSPAR since 2008. He received Science and Engineering Award of the year 2014 from the minister of the ministry of Science Education of Japan and also received the Bset Paper Award of the year 2012 of IJACSA from Science and Information Organization: SAI. In 2016, he also received Vikram Sarabhai Medal of ICSU/COSPAR and also received 20 awards. He wrote 34 books and published 520 journal papers. He is Editor-in-Chief of International Journal of Advanced Computer Science and Applications as well as International Journal of Intelligent Systsems and Applications. http://teagis.ip.is.sagau.ac.jp/ 